[NTERFABEFTECNOLLGGCA

\title{
A CAPTURA DE MOVIMENTO COMO TECNOLOGIA PARA DAR FLUIDEZ NATURAL AOS MOVIMENTOS DE PERSONAGENS E OBJETOS 3D
}

\author{
MOVEMENT CAPTURE AS TECHNOLOGY TO GIVE NATURAL FLUIDITY TO \\ MOVEMENT OF 3D CHARACTERS AND OBJECTS
}

\author{
Saulo Godoy Proetti - sauloproetti@gmail.com \\ Gustavo Henrique Del Vechio - gustavo.vechio@fatectq.edu.br
} Faculdade de Tecnologia de Taquaritinga (Fatec) - Taquaritinga - SP - Brasil

DOI: 10.31510/infa.v17i1.752

\begin{abstract}
RESUMO
A captura de movimento, também chamada por mocap, é uma tecnologia cada vez mais utilizada sobretudo na indústria de jogos e produções audiovisuais, para oferecer mais naturalidade e fluidez ao movimento de objetos e personagens desenhados em 3D. Este estudo se propõe a analisar como esta tecnologia opera, tanto em termos computacionais, isto é, de hardwares e softwares, quanto em sua aplicação prática, mais especificamente em produções cinematográficas. Para isso traz, como exemplo, uma análise do filme "Alita: anjos de combate", em que o uso desta tecnologia proporcionou aos personagens tridimensionais maior fluidez e integração com as filmagens e personagens reais das cenas. $\mathrm{O}$ que se conclui, a partir do estudo, é que sem a captura de movimento, seria muito mais difícil obter tamanho realismo ou fluidez natural no desenvolvimento de animações e artes tridimensionais.
\end{abstract}

Palavras-chave: Captura de movimento. Animação 3D. Computação gráfica (CG).

\begin{abstract}
Motion capture, also called mocap, is a technology increasingly used mainly in the games and audiovisual production industry, to offer more naturalness and fluidity to the movement of objects and characters designed in 3D. This study aims to analyze how this technology operates, both in computational terms, that is, in hardware and software, and in its practical application, more specifically in cinematographic productions. For that, it brings, as an example, an analysis of the film "Alita: combat angels", in which the use of this technology provided the threedimensional characters with greater fluidity and integration with the filming and real characters of the scenes. What is concluded, from the study, is that without the capture of movement, it would be much more difficult to obtain such realism or natural fluidity in the development of animations and three-dimensional arts.
\end{abstract}

Keywords: Motion capture. 3D animation. Computer graphics (CG). 


\section{INTRODUÇÃO}

Este estudo tem o objetivo de avaliar as bases da tecnologia de captura de movimento (mocap), demonstrando sua aplicação sobretudo na indústria cinematográfica, como um recurso eficiente e cada vez mais necessário para se desenvolver animações mais fluídas e naturais de personagens ou objetos tridimensionais.

Para alcançar este objetivo, a metodologia do estudo se define, primeiramente, pela revisão de livros, revistas e matérias especializadas, a fim de compreender o que é a captura de movimento, seus tipos, hardwares e softwares utilizados, além de apresentar alguns exemplos de aplicações reais desta tecnologia em produções cinematográficas. Em seguida, a fim de complementar os conceitos de uso prático, o trabalho volta-se a uma análise de uma produção cinematográfica em particular, que é o filme "Alita: anjos de combate", em que a captura de movimento foi utilizada amplamente para dar mais fluidez aos personagens 3D e integrá-los com mais naturalidade às cenas de filmagens com personagens humanos. A escolha desse filme é intencional por parte dos autores e deve-se pelo fato de ser uma produção recente e com ótima repercussão em relação justamente à tecnologia 3D e de captura de movimento empregadas.

O estudo se justifica pela notação de que, cada vez mais, a tecnologia de captura de movimento é uma realidade acessível a profissionais de diferentes setores, sobretudo, na indústria de jogos (entretenimento) e de produções audiovisuais.

\section{FUNDAMENTAÇÃO TEÓRICA}

A captura de movimento, também conhecida por mocap, é uma técnica de computação gráfica que tem, como principal objetivo, dar vida e fluidez natural aos movimentos de objetos ou personagens desenhados em um computador. ${ }^{1}$ Este é um recurso extremamente útil pois, como explica Junior (2017), "se não existir animações que rodem de forma fluida e natural, isso pode significar um fracasso na aplicação desse efeito [em produções 3D] e, consequentemente, causar uma má impressão e um desconforto visual ao usuário final.”

\footnotetext{
${ }^{1}$ Além de ganhar mais naturalidade em animações, a técnica pode ainda ser usada para simular o rejuvenescimento facial e até reconstruir faces de pessoas já falecidas.
} 


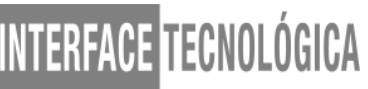

Hoje existem diversos hardwares, tais como roupas especiais equipadas com vários sensores, que podem registrar, de forma precisa, os movimentos naturais de um ser humano e, por meio de softwares especializados, transferir tais informações a objetos ou imagens virtuais. Como exemplo, pode-se citar o uso dessa tecnologia pelo ator Andy Serkis, que dá vida a um chimpanzé com expressões e sentimentos no filme "Planeta dos Macacos", ou ainda Benedict Cumberbatch, que dá vida ao dragão Smaug no filme "O Hobbit". Tais resultados, isto é, a animação fluída desses personagens tridimensionais, seriam muito mais difíceis de serem alcançados sem o uso dos recursos de captura de movimento.

Helbig et al. (2012) explicam que embora os principais campos da captura de movimento incluam os jogos e o cinema, esta tecnologia também pode ser encontrada em diversas outras áreas, tais como nos esportes, em que é possível coletar dados de jogadores quanto a velocidade que eles atingem ao correr, ou ainda a quilometragem que eles percorrem. Além disso, também é possível recorrer a esta tecnologia para realizar simulações de desastres naturais e assim prever ocorrências futuras, bem como projetar automóveis e testá-los em realidade virtual. De maneira geral, aliás, sensores que contam passos em exercícios físicos também podem ser considerados como um tipo de captura de movimento.

\subsection{História do conceito de captura de movimento}

Embora a captura de movimento esteja cada vez mais presente na indústria e possa parecer uma tecnologia de última geração, vale reconhecer que o precursor desta tecnologia remonta há décadas, mais especificamente o começo do século XX. Entre os anos de 1910 a 1940, Fossatti (2009, p. 4) lembra que a animação passava por um intenso processo de industrialização, logo, os curtos prazos, aliados aos altos custos das produções, estimularam os artistas a desenvolverem novas técnicas de desenho. Foi assim que, de acordo com Lucena Jr. (2005, p. 69), a técnica chamada rotoscopia foi criada (figura 1). Isto ocorreu em 1915, a partir dos experimentos de Max e Dave Fleischer, que foram, inclusive, os idealizadores de desenhos icônicos como Popeye, Betty Boop e o palhaço Koko. A invenção dos irmãos Fleischer procurava aprimorar os movimentos dos desenhos animados, cobrindo-lhes de realismo e abrindo novas possibilidades capazes de evoluir e expandir o mercado de animações.

Guillén (1997) acrescenta que o início do século XX também marcou o progresso do cinema, época em que surgiram personagens até hoje conhecidos como Mickey, Gato Félix e 


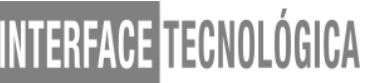

Betty Boop. De acordo com uma matéria no site Animação S.A. (BRANCA..., 2015), o Estúdio Disney, conhecido pelas suas grandes animações desde os anos 1930, foi um dos primeiros a trabalhar com a rotoscopia, mas inicialmente utilizando-a como referência e não para cópia da movimentação de atores. Porém, os animadores excederam esta regra quando, durante a produção do longa animado "Branca de Neve e os sete anões", resolveram utilizar a técnica nas partes mais difíceis, que eram os movimentos fluídos dos personagens humanos.

Figura 1. Criação de uma ilustração por meio da técnica de rotoscopia.

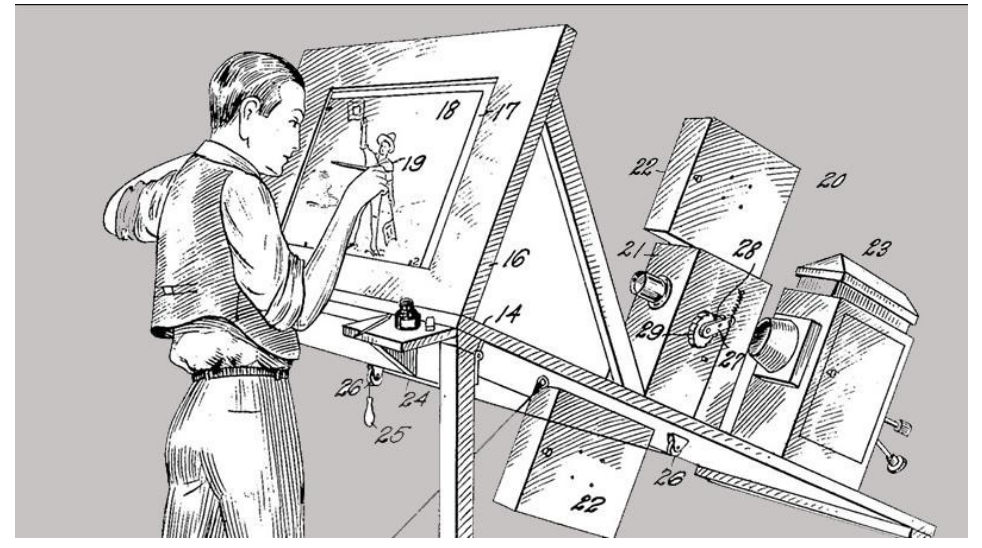

Fonte: site Pluralsight (THIS MONTH..., 2014).

Algumas das grandes animações com rotoscopia ao longo da história incluem, além do já citado desenho "Branca de Neve e os sete anões" (de 1937), o longa-metragem "As viagens de Gulliver", que foi produzido por Max e Dave Fleischer em 1939 (AS AVENTURAS..., s.d), ou ainda o bem mais recente "Loving Vicent", que foi pintado totalmente a óleo em homenagem ao pintor Van Gogh e lançado em setembro de 2017 (LINHARES, 2017).

Como se pode verificar, a rotoscopia ainda hoje é uma tecnologia usada em alguns filmes, embora não se possa deixar de notar que seu uso agora esteja mais voltado ao propósito de recortar atores e inseri-los em outras cenas, o que permite alterar o plano de fundo. ${ }^{2}$ Seja como for, a própria rotoscopia já se tornou automatizada por softwares de edição de imagens, não sendo mais necessário contornar quadro a quadro atores em cena, como foi o caso do filme “A Scanner Darkly”, produzido e lançado em 2006.

\footnotetext{
${ }^{2}$ O Adobe After Effects, popular aplicativo para edição de vídeos, possui uma ferramenta de recorte chamada Rotoscopia, em homenagem a essa técnica do início do século XX.
} 


\title{
2.2 Evolução da captura digital
}

O ator e cineasta britânico Andy Serkis, em entrevista para o canal B9, explica como foi a evolução da captura de movimentos desde 2001, quando trabalhou no filme "O Senhor dos Anéis", interpretando o personagem "Gollum", até seus últimos trabalhos como "Cesar" na trilogia Planeta dos Macacos. Em suas palavras: ${ }^{3}$

\begin{abstract}
Quando filmamos O Senhor dos Anéis tínhamos que filmar tudo duas vezes, primeiro em película, usávamos película naquela época. E então voltar para um pequeno estúdio que se chamava "espaço de captura de movimento". E repetir tudo usando uma câmera 360 graus. E isso mapeava meus movimentos. Durante os próximos filmes e da próxima década fomos capazes de usar captura facial o que não foi possível ser feito em O Senhor dos Anéis. Então isso capturava as expressões faciais do ator e aplicava diretamente a máscara digital. Então fomos capazes de nos movimentar e atuar em qualquer lugar dentro do estúdio de captura. Porque nós tínhamos câmeras nas nossas cabeças, então só estávamos restritas as câmeras ao nosso redor. E então de Planeta dos macacos: A Origem para frente pudemos levar todas essas tecnologias para as locações e sets e filmar esse filme com os atores não sendo restritos pela tecnologia. E a técnica que evoluiu pelo lado da pós-produção é que a renderização e o trabalho artístico que se tem em pegar nossas atuações e colocar nos rostos dos macacos aumentou incrivelmente. A textura da pele, pelo, olhos, a neve no pelo, a água no pelo tudo isso e o software que foi criado para simular essas coisas melhorou radicalmente. Então acho que agora atingimos o ponto onde é o melhor, em termos da atuação dos atores é a melhor representação do que o ator está realmente fazendo, em um personagem de CG (SERKIS, 2017).
\end{abstract}

É importante destacar, no ambiente da captura de movimento, o conceito de "estranheza" em resultados de aplicação desta tecnologia. De acordo com Mori (2012), trata-se da reação observada nas pessoas em relação aos robôs que parecem e agem como seres humanos, mas perceptivelmente não o são. Em outras palavras, a ideia de "estranheza" faz perceber que quando certos jogos, filmes e até mesmo a robótica tentam replicar características humanas, algo nessa aparência pode fazer com muitos expectadores sintam certo desconforto, mesmo sem querer, ao tentar relacionar os personagens com a vida real. Como exemplo, um dos filmes que ilustram esse aspecto é o "Rogue One: uma história Star Wars", que recriou digitalmente os rostos dos atores Peter Cushing interpretando "Moff Tarkin" e Carrie Fisher

3 Entrevista extraída do canal B9. Vide: https://www.youtube.com/watch?v=RMd52GoKQUQ. 
interpretando "Leia". Apesar de muita tecnologia empregada, é perceptível que eles não são, de fato, os atores reais (figura 2).

Figura 2. Camadas de detalhe do processo de computação gráfica.

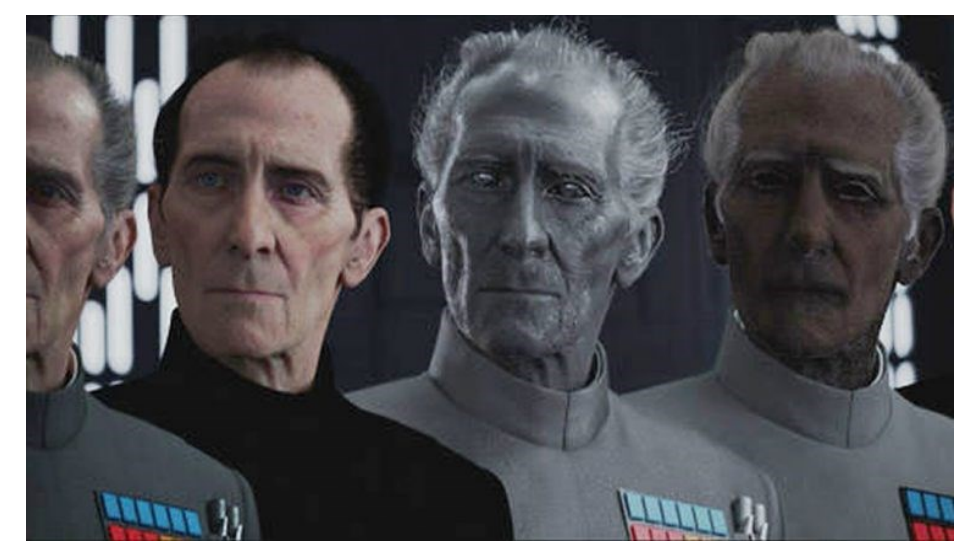

Fonte: Gaglioni (2016).

Há, entretanto, algumas obras que conseguem contornar esse problema: geralmente são as produções que buscam caracterizar os personagens humanos de forma mais cartoon, como visto nos filmes “As Aventuras de Tintim” ou em "Os Incríveis".

\subsection{Técnicas de captura de movimento}

Existem algumas técnicas para a realização da captura de movimento. $\mathrm{Na}$ "captura com marcadores" (figura 3), os movimentos são registrados por, no mínimo, duas câmeras posicionadas para obter a localização dos pontos em relação às coordenadas X, Y e Z. Esses pontos são feitos de um material que reflete a luz das câmeras e ficam espalhados no corpo do ator para que, posteriormente, seja passado para um boneco digital, possibilitando a animação com menor custo e tempo, além de mais fluidez (CONSCIÊNCIA... 2016). 
Figura 3. Andy Serkis, como Gollum, utilizando a captura com marcadores.

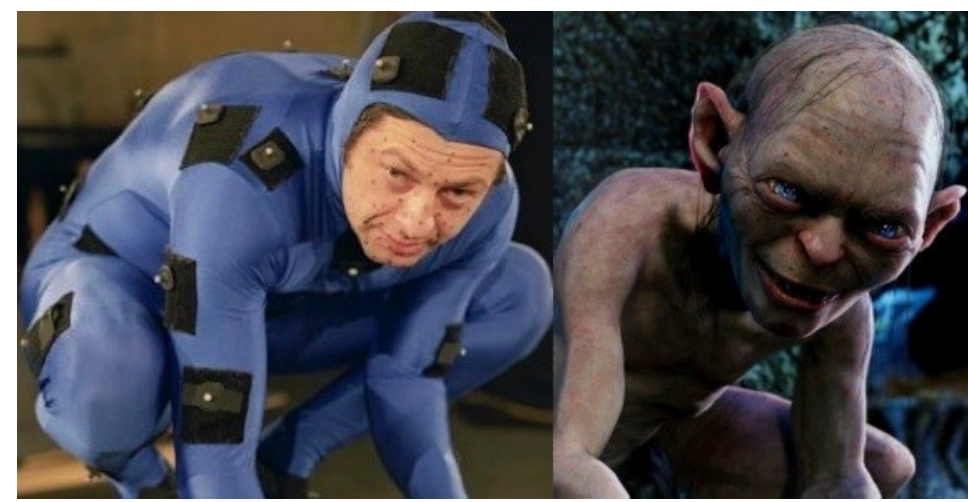

Fonte: site Garotas Geeks (14 GAMES..., 2016).

Já a captura de movimento com sensores, que é conhecida como "Inertial Motion Capture Systems" (figura 4), é baseada em modelos biomecânicos e algoritmos de fusão de sensores. Os sensores, presentes em cada módulo anexado ao corpo, são giroscópio, magnetômetro e acelerômetro, capazes de gerar informações tridimensionais de movimentação que são, posteriormente, aplicadas em objetos ou modelos virtuais (INERTIAL..., 2020).

Figura 4. Exemplo da utilização de captura de movimento com sensores.

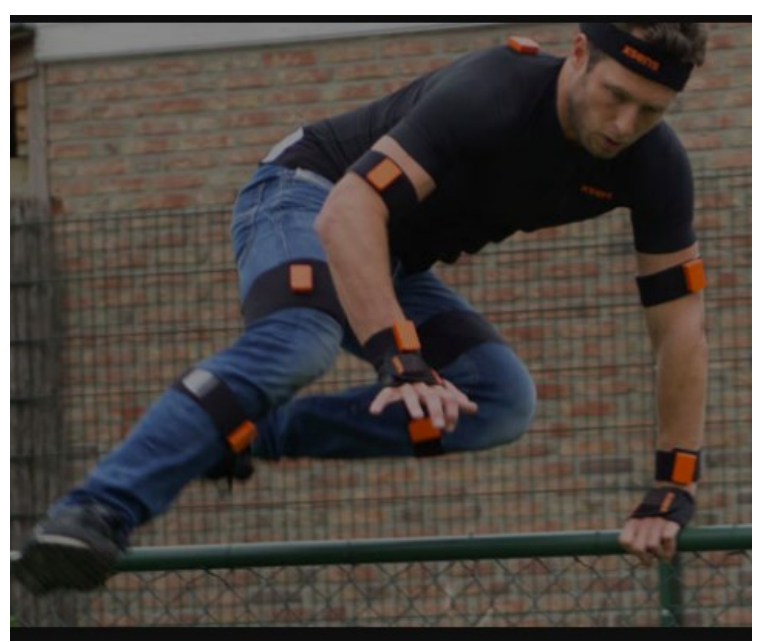

Fonte: Tracklab (INERTIAL..., 2020).

\subsection{Softwares e hardwares}

É muito interessante observar que embora o cinema se aproveite da captura de movimento com hardwares especializados, esta tecnologia está cada vez mais acessível no 
mercado, a profissionais dos mais variados segmentos. Em outras palavras, aplicativos como o Adobe After Effects, Blender, Autodesk Maya ou o Autodesk MotionBuilder ${ }^{4}$ (apenas para citar alguns), já possuem suporte nativo e ferramentas específicas para operar com a captura de movimento. Atualmente, para se rodar estes aplicativos, é recomendado entre 8 GB e 16 GB de memória RAM, um processador com 2 núcleos de 2 Ghz e uma placa de vídeo com 2 GB de VRAM, configurações essas que são, atualmente, acessíveis a muitos profissionais. Ou seja, o que se quer dizer é que não é preciso um supercomputador, somente acessível a grandes empresas, para se trabalhar com a captura de movimento.

Até mesmo os hardwares ou dispositivos, tais como sensores e roupas específicas, estão cada vez mais acessíveis: por exemplo, no site da Enflux (https://bit.ly/39M0amI), empresa especializada neste tipo de produto, uma roupa com sensores para a captura de movimento custa em torno de 900 dólares (há alguns anos, o valor era quase o triplo). Ou seja, esta é uma tecnologia cada vez mais recorrente em vários segmentos, cujo custo de utilização têm se tornado cada vez mais acessível aos profissionais.

Tendo compreendido, portanto, o que é a captura de movimento e sua utilização sobretudo no cinema para dar vida a personagens tridimensionais, apresenta-se, a seguir, uma análise do recente filme "Alita: anjo de combate", lançado em 2019, que usou massivamente este recurso para o sucesso em suas excelentes animações.

\section{PROCEDIMENTOS METODOLÓGICOS}

Escrito por James Cameron e Laeta Kalogridis e dirigido por Robert Rodriguez, o filme “Alita: anjo de combate", é baseado na obra chamada "Gunnm”, do autor Yukito Kishiro. Em termos gerais, Alita (personagem principal) é uma ciborgue reativada, mas que não consegue se lembrar da sua vida passada, e vai em busca de respostas sobre quem ela é.

De acordo com Hellard (2019), a captura facial da atriz Rosa Salazar utilizou duas câmeras, sendo que, para capturar as informações de seu corpo, uma roupa foi desenvolvida especialmente com o intuito de registrar até mesmo os movimentos da sua respiração. Seymour (2019) complementa que vários testes foram feitos com câmeras que ficavam posicionadas na lateral e na frente do rosto da atriz, porém, os diretores perceberam que seria necessário câmeras

\footnotetext{
${ }^{4}$ O Autodesk MotionBuilder é um aplicativo dedicado exclusivamente à animação de personagens por meio de dados obtidos por técnicas de captura de movimento.
} 
dos dois lados do rosto; no final, eles estabeleceram o uso de duas câmeras na frente do rosto, com alimentação de câmera infravermelha (figura 5). Para não perder nenhuma das emoções corporais, os diretores fizeram um boneco digital parecido com a atriz, a fim de que a equipe pudesse estudar os detalhes entre boneco digital e a atuação ao vivo da atriz para, posteriormente, aplicar a atuação no boneco tridimensional de Alita.

Figura 5. Roupa especial e câmeras para a captura de movimento e aplicação em Alita.

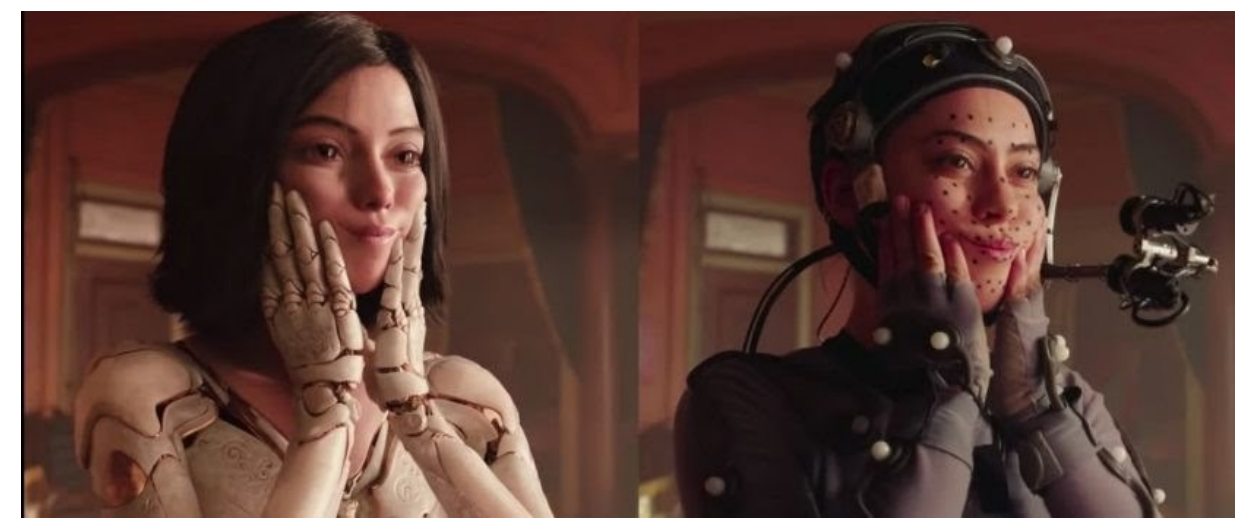

Fonte: Seymour (2019).

Polígonos são usados para gerar objetos ou qualquer detalhe em cenas tridimensionais (é, em outras palavras, a base da criação 3D). Algo que chama muito a atenção para o filme "Alita: anjos de combate" é a complexidade de sua personagem principal: Hellard (2019) descreve que somente os olhos de Alita possuem 9 milhões de polígonos em cada olho, o que é um valor incrivelmente alto para um componente 3D (em termos comparativos, o famoso personagem Gollum, em "O Senhor dos Anéis”, tem cerca de 150 mil polígonos no corpo todo). Isto gerou detalhes tão hiper-realistas que é quase impossível diferenciar seus olhos tridimensionais de uma imagem de um olho real.

Na verdade, os exageros não param por aí: os animadores foram tão perfeccionistas que procuraram reproduzir, com o máximo de detalhes, até mesmo os pelos por todo o corpo da personagens; são incríveis 132.000 pelos na cabeça, 2.000 pelos na sobrancelha, 480 cílios e quase 500.000 pelos pelo rosto e orelhas (HELLARD, 2019).

Figura 6. Detalhes nos olhos da personagem Alita. 


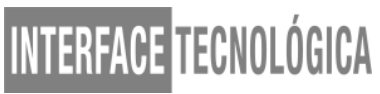

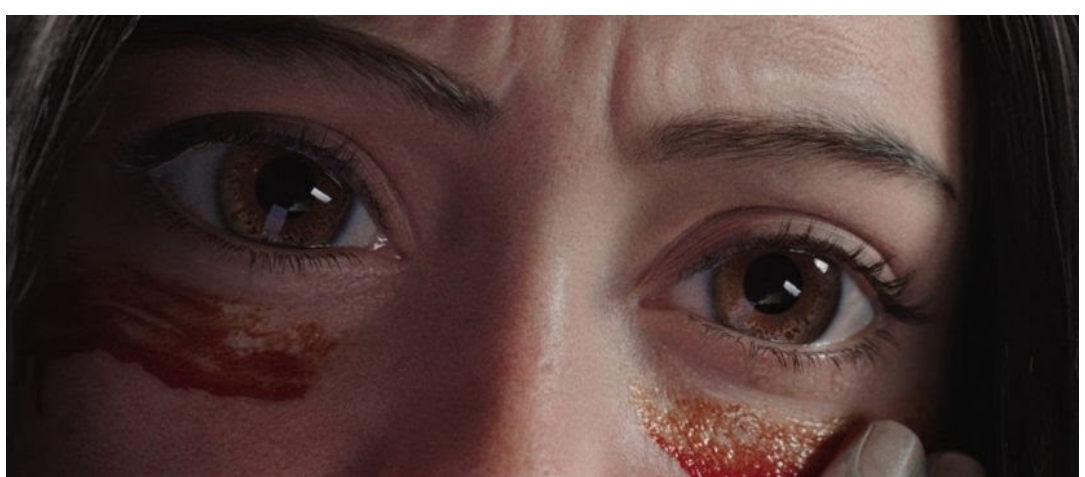

Fonte: Extraído de Seymour (2019).

É evidente que tamanha complexidade 3D exige um hardware com alto poder computacional e preparado para lidar com o cálculo de cada um desses detalhes. Em termos de softwares, a equipe de animação deste filme utilizou-se principalmente do Autodesk Maya, em conjunto com alguns outros softwares proprietários. O filme teve orçamento de 170 milhões de dólares e a complexidade desejada para os personagens foi tanta que, no final, foram necessárias 432 milhões de horas de renderização para concretização da produção!

\section{RESULTADOS E DISCUSSÃO}

O que se percebe, em cada cena do filme "Alita: anjos de combate", é um incrível realismo tanto nos aspectos tridimensionais quanto nos movimentos fluídos dos personagens, fruto de um trabalho dedicado com a tecnologia de captura de movimento. Poucas estranhezas (vide conceito de "estranheza") são percebidas nos movimentos dos membros corporais, sobretudo das expressões facionais, o que inclui a fala, movimento dos olhos etc. Para o crítico de cinema Raphael Santos: ${ }^{5}$

\footnotetext{
...mais linda ainda é toda a computação gráfica do filme. O CGI do filme é impressionante, os efeitos especiais nos efeitos visuais são perfeitos. Alita está interagindo com atores reais e a renderização dela e a qualidade dos movimentos, a integração com o cenário é impressionante desde o primeiro minuto, quando tem um close-up no olho e na pele: é extremamente real chega até assustar!
}

\footnotetext{
${ }^{5}$ Vide crítica de Raphael Santos em: https://www.youtube.com/watch?v=S8eYcKYnSiM
} 
As bilheterias de cinema, que arrecadaram cerca de 405 milhões de dólares, compensaram os 170 milhões investidos nesta obra e as incríveis 432 milhões de horas para renderização total do filme, que justificam tamanha complexidade da produção e, por certo, valem o índice de 92\% de aprovação por parte do público (ALITA..., s.d.). Sem a tecnologia de captura de movimento, dificilmente este longa metragem alcançaria tamanha complexidade nos movimentos, a fim de um personagem tridimensional ser capaz de interagir com cenas de filmagens reais (isto é, com personagens reais) de maneira fluída e natural.

\section{CONSIDERAÇÕES FINAIS}

A tecnologia de captura de movimento é cada vez mais utilizada em produções cinematográficas e, obviamente, têm evoluído constantemente desde quando era feita no papel por técnicas manuais de rotoscopia. Hoje esta tecnologia, além de muito mais acessível a profissionais de diversos setores, permite que diretores, roteiristas e produtores obtenham uma fluidez mais natural nos movimentos de seus objetos e personagens tridimensionais, tendo a possibilidade, inclusive, de misturá-los com atores reais sem que tal fusão deixe muito perceptível o que é real e o que é 3D (em termos de fluidez de movimento). O filme "Alita: anjos de combate" é apenas um exemplo de muitas produções que hoje se aproveitam desta tecnologia, a fim de gerar produções que, de tão complexas e realistas, chegam a assustar.

Além disso, os hardwares e softwares para captura de movimento e aplicação dos dados coletados em objetos e personagens 3D estão mais acessíveis a diferentes segmentos e profissionais. Já existem empresas especializadas em desenvolver dispositivos específicos para esta finalidade (como é o caso da citada Enflux), bem como existem aplicativos que, ou oferecem alto suporte à esta tecnologia, como é o caso do Autodesk Maya, ou são dedicados exclusivamente para esta finalidade, como é o caso do Autodesk MotionBuilder. Sendo assim, a captura de movimento, além de ser essencial em grandes produções, está se tornando uma realidade cada vez mais acessível ao público. É, afinal, uma das grandes tecnologias, emergentes na geração atual da indústria cinematográfica e audiovisual.

\section{REFERÊNCIAS}

14 GAMES que utilizam captura de movimento para deixar os personagens mais realistas. Garotas Geek, 19 abr. 2016. Disponível em: < https://bit.ly/3alHu8t >. Acesso em: 6 fev. 2020. 
ALITA: Battle Angel. Rotten Tomatoes, sd. Disponível em: < https://www.rottentomatoes.com/m/alita_battle_angel >. Acesso em: 2 abr. 2020.

AS AVENTURAS de Gulliver. IMDB, sd. Disponível em: < https://www.imdb.com/title/tt0031397/ >. Acesso em: 15 fev. 2020.

BRANCA de Neve e os sete anões: o filme na história do cinema de animação. Animação S.A, 2 ago. 2015. Disponível em: < https://bit.ly/2UFR2LW >. Acesso em 18 fev. 2020.

CONSCIÊNCIA: captura de movimento. TV UFSC, Youtube, 27 nov. 2016. Disponível em: $<\underline{\text { https: } / / \text { www.youtube.com/watch?v=M gLaLMXkzs }}>$. Acesso em: 27 fev. 2019.

FOSSATI, Carolina L. Cinema de animação: uma trajetória marcada por inovações. VII Encontro Nacional de História da Mídia, Fortaleza, 19-20 ago. 2009. Disponivel em < https://bit.ly/39KX9Tj >. Acesso em: 3 abr. 2020.

GAGLIONI, Cesar. Rogue One: produtores falam sobre CGI e a presença de Tarkin no filme. Jovem Nerd, 27 dez. 2016. Disponível em: $<$ https://jovemnerd.com.br/nerdbunker/rogue-oneprodutora-comenta-presenca-de-tarkin-no-filme/ >. Acesso em: 3 abr. 2020.

GUILLÉN, José M. El cine de animación: en más de 100 longametrajes. Madri: Alianza, 1997.

HELBIG, Henrique; ROSA, Aquila P.; SILVA, Ben-Hur; VARALLO, Daniel; TRIGO, Fabiana; ESPINDOLA, Stephanie. Captura de movimento: nova técnica reinventa a forma de fazer filmes. Identidade RP, 28 jul. 2012. Disponível em: < https://www.rrpponline.com.br/site/captura-de-movimento/ >. Acesso em: 10 mar. 2020.

HELLARD, Paul. Weta Digital VFX supervisor talks about polygons and eyeballs on Alita: Battle Angel. VFX Science, 18 fev. 2019. Disponível em: < https://vfxscience.com/2019/02/18/alita-battle-angel// >. Acesso em: 23 mar. 2020.

INERTIAL Motion Capture Systems. Tracklab, s.d. Disponível em: < https://tracklab.com.au/inertial-motion-capture-systems/ >. Acesso em: 19 fev. 2020.

JUNIOR, Jonas. Por trás das câmeras: o que é a captura de movimento? Cinéfilos Anônimos, 29 jun. 2017. Disponível em: < http://www.cinefilosanonimos.com.br/por-tras-das-cameras-oque-e-captura-de-movimento/ >. Acesso em 15 mar. 2020.

LINHARES, Caroline. Filme sobre a vida de Van Gogh onde todos os frames foram pintados a óleo. O Palma, 22 jun. [2017]. Disponível em: < https://bit.ly/2zaWcoM >. Acesso em: 15 fev. 2020.

LUCENA JÚNIOR, Alberto. Arte da animação: técnica e estética através da história. 2 ed. São Paulo: Senac, 2005. 
MORI, Masahiro. The uncanny valley: the original essay by Masahiro Mori. IEEE Spectrum, 12 jun. 2012. Disponível em: < https://bit.ly/2wZEmqc >. Acesso em: 22 fev. 2020.

SEYMOUR, Mike. Weta Digital's Remarkable Face Pipeline: Alita Battle Angel. FX Guide, 8 mar. 2019. Disponível em: < https://www.fxguide.com/fxfeatured/weta-digitals-remarkableface-pipeline-alita-battle-angel/ > . Acesso em: 23 mar. 2020.

SERKIS, Andy. Andy Serkis explica a evolução da captura de movimentos no cinema. Canal B9, 5 ago 2017. Disponível em: < https://www.youtube.com/watch?v=RMd52GoKQUQ >. Acesso em: 22 fev. 2020.

THIS MONTH in VFX History: rotoscoping and Max Fleischer. Pluralsight, 31 jul. 2014. Disponível em: < https://bit.ly/3dSQ9Y1 $>$. Acesso em 17 mar. 2020. 